\title{
On the Analyticity of Solutions in the Dynamical Mean-Field Theory
}

\author{
Th. Pruschke ${ }^{a}$, W. Metzner ${ }^{b}$, and D. Vollhardt ${ }^{c}$ \\ ${ }^{a}$ Institut für Theoretische Physik, Universität Regensburg, 93040 Regensburg, Germany \\ ${ }^{b}$ Institut für Theoretische Physik C, Technische Hochschule Aachen, 52056 Aachen, Germany \\ ${ }^{c}$ Theoretische Physik III, Elektronische Korrelationen und Magnetismus, Universität Augsburg, 86135 Augsburg, Germany
}

\begin{abstract}
The unphysical solutions of the periodic Anderson model obtained by H. Keiter and T. Leuders [Europhys. Lett., 49, 801 (2000)] in dynamical mean-field theory (DMFT) are shown to result from the author's restricted choice of the functional form of the solution, leading to a violation of the analytic properties of the exact solution. By contrast, iterative solutions of the self-consistency condition within the DMFT obtained by techniques which preserve the correct analytic properties of the exact solution (e.g., quantum Monte-Carlo simulations or the numerical renormalization group) always lead to physical solutions.
\end{abstract}

In a recent paper, Keiter and Leuders [1] discussed the structure and the solutions of the self-consistency condition of the dynamical mean-field theory (DMFT) for the periodic Anderson model at $U=\infty$. They raised two independent points: (i), they showed analytically that on certain assumptions for the structure of the solution the self-consistency condition can have multiple solutions, which merge in the upper half of the complex plane, and (ii), they argued that calculations of $1 / d$ corrections $(d$ : spatial dimension of the lattice) to the self-consistency condition of the DMFT are problematic, since the limit $d \rightarrow \infty$ does not commute with the limit $n \rightarrow \infty$ of the length $n$ of loops embedded in the lattice.

In the following both points will be addressed. The main part of the paper will be concerned with a detailed analysis of the first issue. In particular, we will show that the conclusions of Keiter and Leuders [1] relating to this point are unwarranted, i.e., are due to the deficiency of the method used by these authors to solve (or simulate the solution of) the single-impurity Anderson model (SIAM).

\section{A. Structure of the solution of the self-consistency condition}

Multiple solutions of a nonlinear set of equations are not unusual. In particular, most multiple solutions of nonlinear equations for propagators are unphysical, i.e., have poles in the upper half-plane or lead to an incorrect asymptotic behavior (or both). In this respect the findings by Keiter and Leuders [1] that under certain conditions there exists a branch point in the upper half-plane where an unphysical and the physical solution to the selfconsistency condition merge, is disturbing. If true in gen- eral it would raise doubts about the uniqueness of numerically obtained iterative solutions to the self-consistency condition, and would make the physical relevance of results obtained within the DMFT questionable.

Here we will show that, in fact, the self-consistency condition for the periodic Anderson model (PAM) [2] always leads to a unique physical result unless the functional space for the solutions is artificially restricted as done in ref. [1]. In standard notation the PAM reads

$$
\begin{aligned}
H_{\mathrm{PAM}}= & \sum_{\mathbf{k} \sigma} \epsilon_{\mathbf{k}} c_{\mathbf{k} \sigma}^{\dagger} c_{\mathbf{k} \sigma} \\
& +\sum_{i \sigma}\left(\epsilon_{f}+\frac{U}{2} f_{i \bar{\sigma}}^{\dagger} f_{i \bar{\sigma}}\right) f_{i \sigma}^{\dagger} f_{i \sigma} \\
& +V \sum_{i \sigma}\left(c_{i \sigma}^{\dagger} f_{i \sigma}+\text { h.c. }\right)
\end{aligned}
$$

Within the DMFT, the nontrivial part of the partition function is represented by an effective local action [3]

$$
\begin{aligned}
S_{\mathrm{eff}}= & -\sum_{\sigma} \int d \tau d \tau^{\prime} f_{\sigma}^{\dagger}(\tau) \tilde{\mathcal{G}}^{-1}\left(\tau-\tau^{\prime}\right) f_{\sigma}\left(\tau^{\prime}\right) \\
& +U \int d \tau f_{\uparrow}^{\dagger}(\tau) f_{\uparrow}(\tau) f_{\downarrow}^{\dagger}(\tau) f_{\downarrow}(\tau)
\end{aligned}
$$

where [4

$$
\tilde{\mathcal{G}}(z)=\left[z-\epsilon_{f}-V^{2} \mathcal{G}(z)\right]^{-1}
$$

and the auxiliary propagator $\mathcal{G}(z)$ describes an artificial system where the $f$-states at the site under consideration have been removed. Note that the action (2) defines an effective SIAM with band-electron Green function $\mathcal{G}(z)$ which has to be determined self-consistently. For the PAM, (11), Keiter and Leuders derived the following expression [1]:

$$
\mathcal{G}(z)=\frac{1}{N} \sum_{\mathbf{k}} \frac{g_{\mathbf{k}}(z)}{1-g_{\mathbf{k}}(z) T(z)+T(z) \mathcal{G}(z)} .
$$

Here $g_{\mathbf{k}}(z)=1 /\left(z-\epsilon_{\mathbf{k}}\right)$ is the propagator of the noninteracting conduction band system and $T(z)=V^{2} F(z)$, with $F(z)$ as the local $f$-electron Green function. The latter relation implies that $T(z)$ cannot be chosen at liberty. Eq. (4) is not sufficient to solve the problem, since $F(z)$ is unknown. The quantity $F(z)$ has to be determined from an effective SIAM, where the propagator of the host electrons is given by $\mathcal{G}(z)$. It should be noted 
that the relation (4) is not only valid for $U=\infty$, but for all values of $U$, including $U=0$. Moreover, it is important to mention that in Ref. [1] the artificial host propagator $\mathcal{G}(z)$ appearing in (3) is denoted by $G(z)$, while conventionally, and also in this paper, $G(z)$ refers to the propagator of the physical band states.

Let us briefly comment on the validity of Equ. (4) for $U=0$. At first glance it seems rather strange that the solution to a non interacting system should be given by a complicated, non-linear self-consistency condition. The reason, however, is quite simple. First, Equ. (4) is set up in real space, while the solution to (11) for $U=0$ is naturally obtained in reciprocal space. Second, as mentioned before, the quantity $\mathcal{G}(z)$ is not a physical propagator but an artificial auxiliary quantity. It is perfectly valid, although not necessarily reasonable, to rewrite the solution of the PAM (11) in terms of this auxiliary local propagator even for $U=0$, thus obtaining the complicated expression (4). For the physical propagator $G(z)$, on the other hand, one eventually finds, inserting $T(z)=V^{2}\left[z-\epsilon_{f}-V^{2} \mathcal{G}(z)\right]^{-1}$, a simple and sensible solution (cf. Equ. (7) below). It must be noted that for $U=0$ this procedure is exact independent of dimension provided the hybridization in (1) is local. The DMFT or limit $d=\infty$ enters at a different stage, namely through the locality of $T(z)$ even in the presence of the two-particle interaction $U$.

To examine the analytic properties of (4) under the condition that $T(z)$ is given by the solution to an effective SIAM some simple algebraic transformations have to be performed. Let us assume that $T(z)$ and $\mathcal{G}(z)$ are solutions to (4). Then we may rewrite (4) as

$$
\mathcal{G}(z)=\frac{1}{1+T(z) \mathcal{G}(z)} \frac{1}{N} \sum_{\mathbf{k}} \frac{1}{z-\epsilon_{\mathbf{k}}-\frac{T(z)}{1+T(z) \mathcal{G}(z)}} .
$$

For the SIAM, the host-electron propagator of the interacting system is obtained from the host propagator of the system without $f$-states by

$$
G(z)=\mathcal{G}(z)+\mathcal{G}(z) T(z) \mathcal{G}(z) .
$$

We can rewrite (4) in terms of the physical host-electron propagator as

$$
G(z)=\frac{1}{N} \sum_{\mathbf{k}} \frac{1}{z-\epsilon_{\mathbf{k}}-\frac{T(z)}{1+T(z) \mathcal{G}(z)}},
$$

and thereby identify the self-energy

$$
\Sigma^{(c)}(z)=\frac{T(z)}{1+T(z) \mathcal{G}(z)}
$$

of the host electrons. Note that for $U=0$ the self energy (8) reduces to the well-known expression $\Sigma^{(c)}(z)=$ $V^{2} /\left[z-\epsilon_{f}\right]$. Since the $\mathbf{k}$-dependence in (7) is only due to $\epsilon_{\mathbf{k}}$, the $\mathbf{k}$-sum can be converted to an energy integral

$$
\frac{1}{N} \sum_{\mathbf{k}} \ldots \rightarrow \int d \epsilon \rho^{(0)}(\epsilon) \ldots
$$

with a positive-definite density of states (DOS) $\rho^{(0)}(\epsilon)$. The relation (7) is simply a Hilbert transform; therefore one can make use of its analytical properties to show [5]

$$
G(z)=\frac{1}{z-\Sigma^{(c)}(z)-\Gamma\left(z-\Sigma^{(c)}(z)\right)},
$$

with a Herglotz-analytic function $\Gamma(\zeta)$, i.e.

$$
\operatorname{sign}(\Im m \Gamma(\zeta))=-\operatorname{sign}(\Im m \zeta)
$$

and $\Gamma(\zeta) \sim 1 / \zeta$ for $|\zeta| \rightarrow \infty$. Furthermore, $\Gamma(\zeta)$ is, in principle, a known function which is determined by the structure of the DOS alone. Finally, $\mathcal{G}(z)$ can be obtained from the definition of $G(z)^{-1}=\mathcal{G}(z)^{-1}-\Sigma^{(c)}(z)$ as

$\mathcal{G}(z)^{-1}=G(z)^{-1}+\Sigma^{(c)}(z)=z-\Gamma\left(z-\frac{T(z)}{1+T(z) \mathcal{G}(z)}\right)$.

It should be stressed that (11) is not merely a nonlinear equation determining $\mathcal{G}(z)$ but, rather, a functional equation, since $T(z)$ is a functional of $\mathcal{G}(z)$. The functional dependence is determined by the solution of a nontrivial problem, namely the SIAM. It is thus not sufficient merely to study the possible roots of the nonlinear equation (11), but one has to discuss the structure of this equation under the constraint that $T(z)$ must obey the basic properties of the solution to the SIAM (2). Since there does not exist an analytic solution to the SIAM in closed form this constraint can be incorporated currently only within an iterative scheme, even if $\Gamma(\zeta)$ is known in closed form as, for example, for the Bethe lattice or the toy DOS used in [1].

Let us therefore assume that we knew the exact solution of the SIAM, and study the consequences of an iterative procedure to solve the self-consistency condition. Starting in the $n$-th step of this iterative procedure with a physical $\mathcal{G}^{(n)}(z)$ with $\operatorname{sign}\left(\Im m \mathcal{G}^{(n)}(z)\right)=-\operatorname{sign}(\Im m z)$ and $\mathcal{G}^{(n)}(z) \sim 1 / z(|z| \rightarrow \infty)$, the corresponding propagator of the $f$-states, $F^{(n)}(z)$, and hence $T^{(n)}(z)$, will have the same properties, i.e., the right-hand side of (11) will definitely have the correct asymptotics, because $T^{(n)}(z) \mathcal{G}^{(n)}(z) \sim 1 / z^{2}$ and thus

$$
\frac{T^{(n)}(z)}{1+T^{(n)}(z) \mathcal{G}^{(n)}(z)} \sim 1 / z
$$

as $|z| \rightarrow \infty$. More importantly, however, the exact solution to the effective SIAM (2) has to satisfy the condition 6

$$
\operatorname{sign}\left[\Im m\left(T^{(n)}(z)^{-1}+\mathcal{G}^{(n)}(z)\right)\right]=+\operatorname{sign}(\Im m z)
$$

which is necessary to ensure that $\Sigma^{(c)}(z)$, (8), has the correct analytic properties, i.e., $\operatorname{sign}\left(\Im m \Sigma^{(c)}(z)\right)=$ $-\operatorname{sign}(\Im m z)$. Under those conditions, the iterative procedure always stays within the subspace of physical solutions. Here it must be stressed once more that one is 
not free to choose $T^{(n)}(z)=V^{2} F^{(n)}(z)$, or even choose a particular functional form for $T^{(n)}(z)$, since this form is entirely and uniquely determined by the solution of the effective SIAM with a fixed host Green function $\mathcal{G}^{(n)}(z)$. Especially condition (12) turns out to be very susceptible to approximations and is easily violated. Such a violation would manifest itself in an indefinite sign of $\Im m \Sigma(z)$ and would thus lead to the crossing of a branch point into the upper half plane. Note also that in the course of the iteration, the quanitities entering on the right-hand side of (11) are those of the $n$-th step, producing the host Green function $\mathcal{G}^{(n+1)}(z)$ for the $n+1$-th step and, unless self-consistency has been reached, $\mathcal{G}^{(n+1)}(z) \neq \mathcal{G}^{(n)}(z)$ in general [7].

Hence we find that the branch point in the upper halfplane observed by Keiter and Leuders [1] is merely due to the author's restricted choice of the functional form of $T(z)$ which leads to a violation of (12). Namely, motivated by results for the standard SIAM these authors chose for $T(z)$ the following ansatz [9]

$$
T(z)=V^{2}\left(\frac{a_{\epsilon_{f}}}{\omega-\epsilon_{f}+i \Gamma_{\epsilon_{f}}}+\frac{a_{\mathrm{K}}}{\omega+i \Gamma_{\mathrm{K}}}\right) .
$$

This form represents the strong-coupling regime of the SIAM, i.e. $-\epsilon_{f}, \epsilon_{f}+U \gg \pi \rho^{(0)}(0) V^{2}$. The original singleparticle level at $\epsilon_{f}$ is broadened by the coupling to the conduction bath. In addition, the sharp Abrikosov-Suhl resonance which characterizes the virtual bound state generated by spin-flip scattering appears at the Fermi energy. A typical result will have the form shown in Fig. 11a. In Fig. 1 we assumed a Gaussian free DOS $\rho^{(0)}(\epsilon)=\exp \left(-\epsilon^{2}\right) / \sqrt{\pi}$ and set $V^{2}=0.2, \epsilon_{f}=-1$. Let us compare this result with a fully self-consistent DMFT calculation for the PAM with the same parameters [8]. The result is shown in Fig. 1 $1 \mathrm{~b}$ (full line). It is obvious from the inset to Fig. 1 $1 \mathrm{~b}$ that the true solution of the PAM does not have the simple structure given by (13) assumed in ref. 11]. In particular, one does not observe a single peak at the Fermi energy, but two peaks separated by a gap.

Consequently, the conclusion following equ. (10) in ref. [1] is unwarranted: the observed breakdown, i.e. the appearance of a branch point in the upper half plane, does not result from the fact that in this regime physically interpretable single-valued solutions cannot be found, but merely from the deficiency of the method used to solve (or simulate the solutions of) the SIAM. In particular, one of the authors [10] and F. Anders [11] observed that approximations based on the resolvent perturbation theory [12], like the non-crossing approximation (NCA) or improved versions of it, always lead to spectra similar to (13) and are not able to reproduce more complicated features like the double-peak structure at the Fermi energy appearing in Fig. 1 $1 \mathrm{~b}$. It is therefore not surprising that the application of these techniques in solving the DMFT for the PAM leads to the breakdown described in ref. [1]: it is a problem generated by the method used to solve the self-consistency condition and not of the self-consistency condition itself.
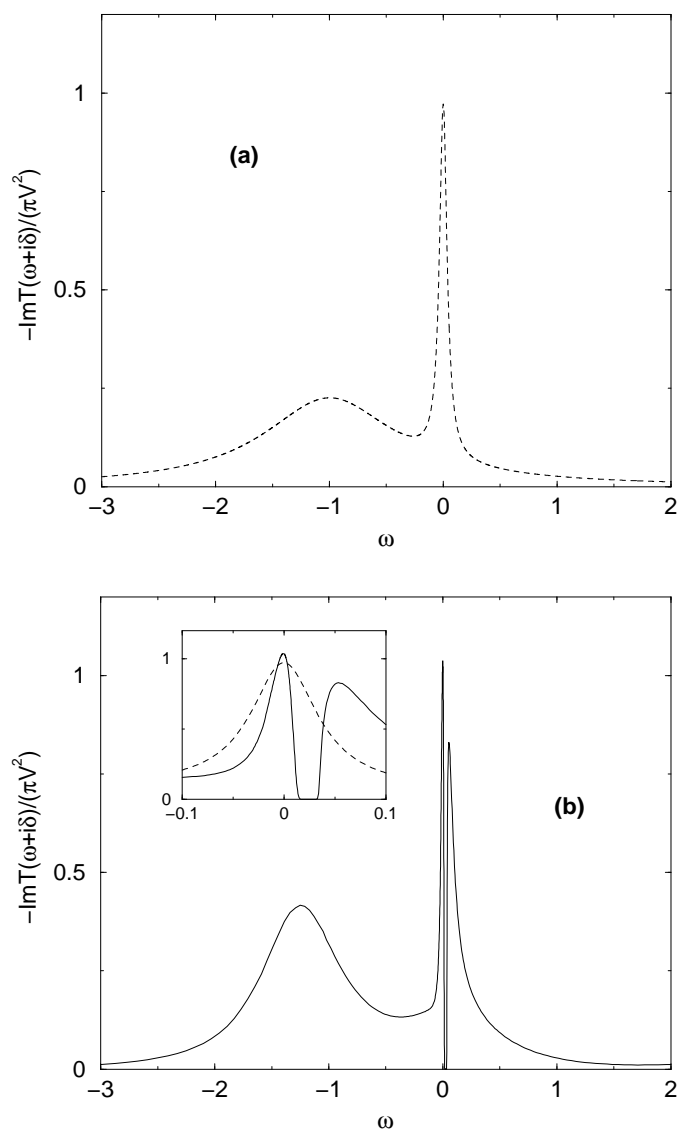

FIG. 1. Imaginary part of $T(\omega+i \delta)$ for the PAM. (a) result according to (13) as used in ref. 1]; (b) result from a full DMFT calculation with the NRG 8]. In the inset in (b) the two curves are compared for energies close to the Fermi energy.

Our arguments do not imply that the self-consistency condition (11) must necessarily have only one unique solution for a given set of model parameters. Such a conclusion would require a more detailed knowledge of the solutions of the SIAM (especially, whether there exists a one-to-one correspondence between $\mathcal{G}(z)$ and $F(z)$ ), and of the extent of the basin of attraction for a particular solution. Since there does not exist an exact analytical solution to the SIAM for an arbitrary host Green function $\mathcal{G}(z)$, statements about these properties of the self-consistency condition are out of reach. Indeed for the Hubbard model such a situation (namely, two physically valid, coexisting solutions) occurs close to the MottHubbard metal-insulator transition [3].

\section{B. $1 / d$ corrections to the DMFT}

In DMFT a fermionic lattice model is mapped onto a SIAM, complemented by a self-consistency condition [3]. 
This mapping is rigorous in $d=\infty$, a fact which is not disputed in ref. [1].

The possibilities and limitations of systematic $1 / d$ expansions around $d=\infty$ have already been explored by a number of authors [13 19]. Whether $1 / d$-corrections to DMFT are able to improve the theory depends on the quantity under investigation, on the type of $1 / d$ expansion, and also on the physics one would like to describe. For example, while $1 / d$-corrections include nonlocal short-range correlations they are unable to capture certain long-range correlations. Furthermore, $1 / d$ corrections, when truncated at finite order, may violate basic analytical properties of dynamical quantities [16]. All this is not unexpected. Indeed, analogous problems arise in $1 / d$-expansions around the Weiss mean-field theory for the Heisenberg model and the coherent potential approximation for the Anderson disorder model [14]. We do not see why the peculiarities of large loops in the large $d$ limit emphasized by Keiter and Leuders [1] should make expansions around DMFT particularly difficult or ambiguous. Since even the Green function of the non-interacting system can be expanded in loops, it is clear that these peculiarities are in one-to-one correspondence with the special behavior of the bare DOS in the limit $d \rightarrow \infty$ (e.g., the absence of van Hove singularities) 20,21. Potential problems with the $1 / d$-expansion of the bare DOS can be avoided by inserting the actual DOS of the finite-dimensional system into the DMFT equations. Then only corrections to the local approximation of irreducible vertices underlying the DMFT need to be considered, as has been done already by several authors [13,18,22, 23].

In summary, we showed that the iterative solution of the self-consistency condition within the DMFT always lead to solutions that stay in the physical subspace. Therefore, the unphysical branch cuts observed by Keiter and Leuders [1] are due to the method used by these authors to solve the SIAM which violates fundamental analytic properties of the exact solution. Similar violations are caused by the NCA and other approximations based on the resolvent perturbation theory [12]. In fact, this also appears to be the reason why it was not possible up to now to obtain meaningful results for the PAM using NCA and related methods, while techniques which preserve the correct analytic properties of the exact solution (e.g., iterated perturbation theory, quantum Monte-Carlo simulations or the numerical renormalization group) do not encounter similar difficulties.

We thank F. Anders, V. Janiš, M. Jarrell, H. Keiter and D. Otto for useful communication.

* Permanent address as of June 1, 2001: Theoretische Physik III, Elektronische Korrelationen und Magnetismus, Universität Augsburg, 86135 Augsburg, Germany
[1] H. Keiter and T. Leuders, Europhys. Lett. 49, 801(2000).

[2] Here we shall focus on the PAM in order to make direct contact with the discussion in ref. [i]. However, the arguments presented are valid also for general one-band models.

[3] A. Georges, G. Kotliar, W. Krauth, M. Rozenberg, Rev. Mod. Phys. 68, 13 (1996).

[4] The appearance of a momentum independent hybridization $V$ in (11) implies that the representation of $\tilde{\mathcal{G}}(z)$ is not unique. Therefore the form of $\tilde{\mathcal{G}}(z)$ used in ref. [1] and here differs from the one used in ref. [3], but can be derived from it using simple algebra.

[5] Th. Maier, M. Jarrell, Th. Pruschke and J. Keller, Eur. Phys. Journal B13, 613(2000).

[6] This relation follows quite simply from the fact that $T(z)=V^{2} F(z)$, where the local propagator $F(z)$ has the form $F(z)=\left[z-\epsilon_{f}-V^{2} \mathcal{G}(z)-\Sigma^{(f)}(z)\right]^{-1}$ with a Herglotz-analytic self-energy $\Sigma^{(f)}(z)$.

[7] One consequence is that, for an iterative solution, (5) is not a valid reformulation of (11), because it only holds when self-consistency has been reached.

[8] Th. Pruschke, R, Bulla and M. Jarrell, Phys. Rev. B61, 12799(2000).

[9] T. Leuders, Ph.D. thesis, University of Dortmund 1998.

[10] T. Pruschke, private communication.

[11] F. Anders, private communication.

[12] H. Keiter and J.C. Kimball, Phys. Rev. Lett. 25, $672(1970)$.

[13] F. Gebhard, Phys. Rev. B41, 9452(1990).

[14] R. Vlaming and D. Vollhardt, Phys. Rev. B 45, 4637 (1992).

[15] R. Vlaming, G. Uhrig and D. Vollhardt, J. Phys.: Condens. Matter 4, 7773(1992); E. Halvorsen, G.S. Uhrig and G. Czycholl, Z. Phys. B94, 291(1994); G.S. Uhrig, Phys. Rev. B54, 10436(1996).

[16] P.G.J. van Dongen, Phys. Rev. B 50, 14016 (1994). It follows immediately from (A9) therein, that the spectral weight has an acausal part of order $\exp (-2 / \sqrt{d})$ [P.G.J. van Dongen, private communication]; P.G.J. van Dongen and T. Schauerte, to be published.

[17] A. Schiller and K. Ingersent, Phys. Rev. Lett $\mathbf{7 5}$, 113(1995); G. Zaránd, D.L. Cox and A. Schiller, condmat/0002073.

[18] M. Hettler, A.N. Tahvildar-Zadeh, M. Jarrell, Th. Pruschke and H.R. Krishnamurthy, Phys. Rev. B58, 7475(1998); M. H. Hettler, M. Mukerjee, M. Jarrell, and H. R. Krishnamurthy, Phys. Rev. B61, 12739 (2000).

[19] V. Janiš and D. Vollhardt, Phys. Rev. B 63, 125112 (2001).

[20] E. Müller-Hartmann, Z. Phys. B 74, 507 (1989).

[21] Indeed, for the Bethe lattice, where self-avoiding loops do not exist, the large-loop peculiarities discussed in Ref. [1] and the unphysical band tails typical for hypercubic lattices in $d \rightarrow \infty$ are absent. Nevertheless the DMFT and (10) is well-defined also for a Bethe lattice.

[22] W. Metzner, Z. Phys. B 77, 253 (1989).

[23] Th. Maier, M. Jarrell, Th. Pruschke, and J. Keller, Phys. Rev. Lett. 85, 1524 (2000). 